\title{
Scaffolding Singaporean Students to Write Vividly in the Chinese 'Mother Tongue', Mandarin
}

\author{
Tzemin Chung \\ School of Education, James Cook University \\ PO Box 6811, Cairns, Queensland, Australia 4870 \\ E-mail: tzemin@gmail.com \\ Neil Anderson (corresponding author) \\ School of Education, James Cook University \\ PO Box 6811, Cairns, Queensland, Australia 4870 \\ E-mail: neil.anderson@jcu.edu.au \\ Munkew Leong \\ Institute of Systems Science, National University of Singapore \\ 21 Lower Kent Ridge Rd, Singapore 119077 \\ E-mail: munkew@gmail.com \\ Waiyin Choy \\ Teacher, Ministry of Education, Singapore
}

Doi:10.7575/aiac.alls.v.5n.1p.40

Received: 05/01/2014

URL: http://dx.doi.org/10.7575/aiac.alls.v.5n.1p.40

Accepted: 26/02/2014

\begin{abstract}
This paper details results from a three-year study investigating how to help students in Singapore write vivid compositions in Mandarin, the Chinese 'mother tongue'. Mastery of the mother tongue by Singaporean students has become an important government priority in recent years. The strategies employed by this study included the use of information and communications technology (ICT) mediated scaffolds such as collaborative mind maps and online peer editing to help students learn micro-writing strategies. This paper outlines the process of using various scaffolds to support students to learn and apply the action chain micro-writing strategy. A class of 31 Primary 4 from a neighbourhood school participated in this study. Findings indicated that students were very enthusiastic about writing in the ICT-mediated environment. Contrary to the teacher's initial belief, students could be scaffolded to write action chains quickly. Findings highlighted the potential of scaffolding students in learning small chunks of writing strategy in an ICT-mediated environment that enabled them to practice these strategies in their composition writing until they could master and apply them. These micro-writing strategies gradually built up to a complex set of skills, including expressive writing. Moreover, students enjoyed working in groups and editing their peers' work online. This showed that peers could be engaged as scaffolders in the classroom to free up the teacher' time, allowing the teacher more time to spend with students who were not performing well.
\end{abstract}

Keywords: collaborative learning, pedagogical issues, elementary education, teaching/learning strategies, applications in subject areas

\section{Introduction}

This paper begins with an overview of the Singaporean 'mother tongue' environment and how it influences the motivation to learn one's 'mother tongue' in neighbourhood schools. The pilot phase of the study and its findings that reflect real world issues in the teaching of Chinese composition writing in these schools are described. It continues to introduce a writing program that aims at scaffolding students to write vivid compositions in Chinese. Both research issues in instructional scaffolding and real world classroom issues are considered in the design of the writing program. The paper further describes how the writing model was introduced in school and the data collected for analysis. Findings of implementing the writing program in a school are discussed. Future refinements of the writing program are also included.

\subsection{The Singapore Context}

Chinese is the second school language for ethnic Chinese and is referred to as a "Mother Tongue" language in the education system in Singapore. Mother tongue is defined as the language learned by children and passed from one generation to the next (wordnet.princeton.edu/perl/webwn) but this is not the case in Singapore where it may not be 
students' home language (Dixon, 2005). Chinese speaking families of Primary One pupils have declined from $90 \%$ in 1980 (Ministry of Education, 2012), to 47.2\% in 2005 (Pakir, 2008). In 2005, 93\% of affluent families (defined as those who live in private condominiums and landed properties) surveyed spoke English or a mix of English and Chinese at home compared to $60 \%$ of families living in 3-room government housing board flats (Zhao, Liu, \& Goh, 2007). Thus, children in Singapore today lack the environment to learn Chinese at home. Hence one of the most significant factors influencing literacy proficiency is absent, specifically, informal contact with native speakers, especially peers and family (Moyer, 2004).

The school environment in Singapore is also not conducive for learning Chinese as students spend only five to eight hours per week taking Chinese lessons. Typically, they would only write one composition per month that is done in a one and a half hour class. Hence the level of composition writing skills in Singapore is generally low. Sim (2005) reported that students had insufficient vocabulary and lacked basic sentence formation skills. They also lacked observation abilities and creative thinking skills. In addition, their vocabulary, grammatical and sentence structures were influenced by Chinese dialects, English, Malay, and Tamil (Lu, Zhang, Qian, 2002).

The Ministry of Education (MOE, 2012) in Singapore embraced information technology as one way to motivate students to write more. On a nation-wide scale, ICT (Information Communications Technology) tools such as edictionaries, Chinese character input systems, and online access to resources were added to the Chinese curriculum to raise the interest in learning Chinese. Furthermore, the MOE intends to enhance these efforts into a complete ICTmediated writing program (Personal interview with a MOE curriculum planning and development officer, June 25, 2007). A summary of the interview includes the following:

1. It is worth putting in effort to develop a complete ICT-mediated writing program. We think a writing package is most needed in primary school;

2. ICT-mediated scaffolding should be a viable approach in helping students write better compositions. Teachers can go into the software and customize scaffolds for students;

3. Look into the development of writing software. We should look into companies to develop software and promote it in school;

4. Enable ICT-mediated peer-critique. Include game-based or reward-based software.

5. How can Chinese be more of a part of the pupils' life? Using ICT may achieve that.

From the interview, we could discern clear areas of interest in the MOE intended efforts such as ICT-mediated scaffolding, peer-critique, games and creating an immersive Chinese environment in and out of school. As part of these efforts, the MOE Curriculum Planning and Development Division for Chinese language was exploring means to incorporate a technology platform into the Chinese language curriculum that can support a comprehensive writing strategy, building of scaffolds, peer-critique, educational games, rewards, and lifelong learning.

\section{The Pilot Study}

In August 2007, a pilot study was conducted in a typical neighbourhood school to explore the possibility of incorporating an ICT-mediated platform to help students write better Chinese compositions. Spilchuk (2009) describes Singaporean neighbourhood schools as "government schools which are in lower socio-economic neighbourhoods, or within government housing developments that are apartment style rather than those government schools which are in 'elite' upper or middle class areas of the city where residents live in condominiums, terrace houses and individual homes" (para. 1). Typically, these schools have large classes of up to 32 students, lack resources, and students in the Chinese classes were of mixed-ability.

In Chinese composition lessons, students are given four pictures to compose a story. They are stories that teach moral values. The minimum word count is 80 . Teachers would typically start the composition lesson with an introduction to the composition topic and highlight ten possible words students could appropriately use in their paper. Students would then write the composition and would often ask the teacher how to transcribe words they needed for their compositions. This usually took up a lot of the class time. Students have to submit their compositions at the end of the lesson. As such, teachers did not have time to help the better students at the idea level or the weak students to construct and flow sentences.

As such, the school felt that they needed to enhance the existing writing instruction to include writing skill training and provide differentiated learning for different ability students. From the beginning of the pilot, it was understood that the training would be conducted within curriculum time so that it could be adopted as part of normal teaching rather than as a one-off special experiment or an after-school enrichment program. The pilot was run between August 2007 and November 2008.

\subsection{The software platform}

The software platform developed for this study was an e-portfolio system called FolioStar (see Figure 1). It was developed for its ease of use and flexibility in customizing scaffolding templates and assigning writing tasks to individual students (see Figure 2) 


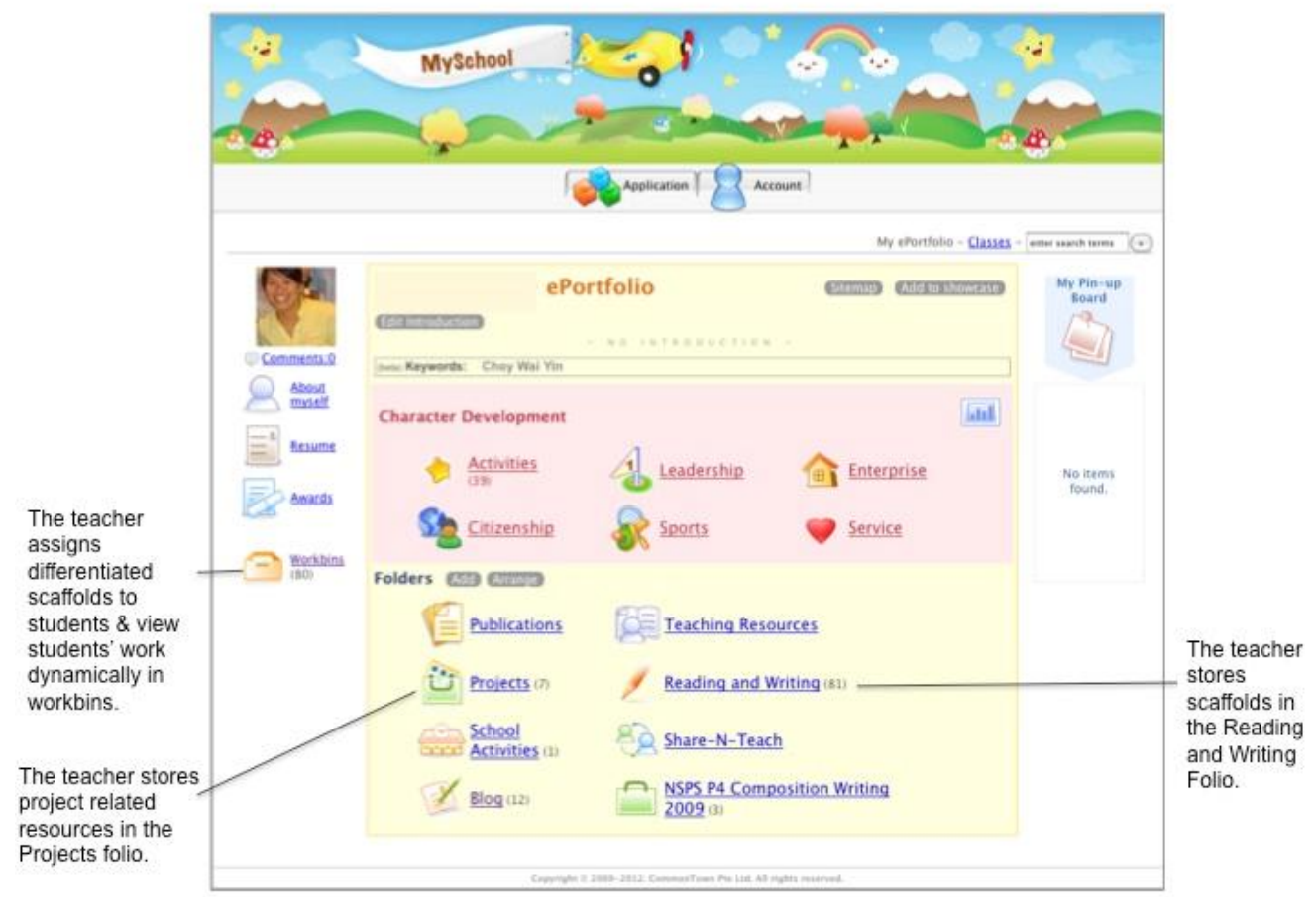

Figure 1. Features of e-portfolio.

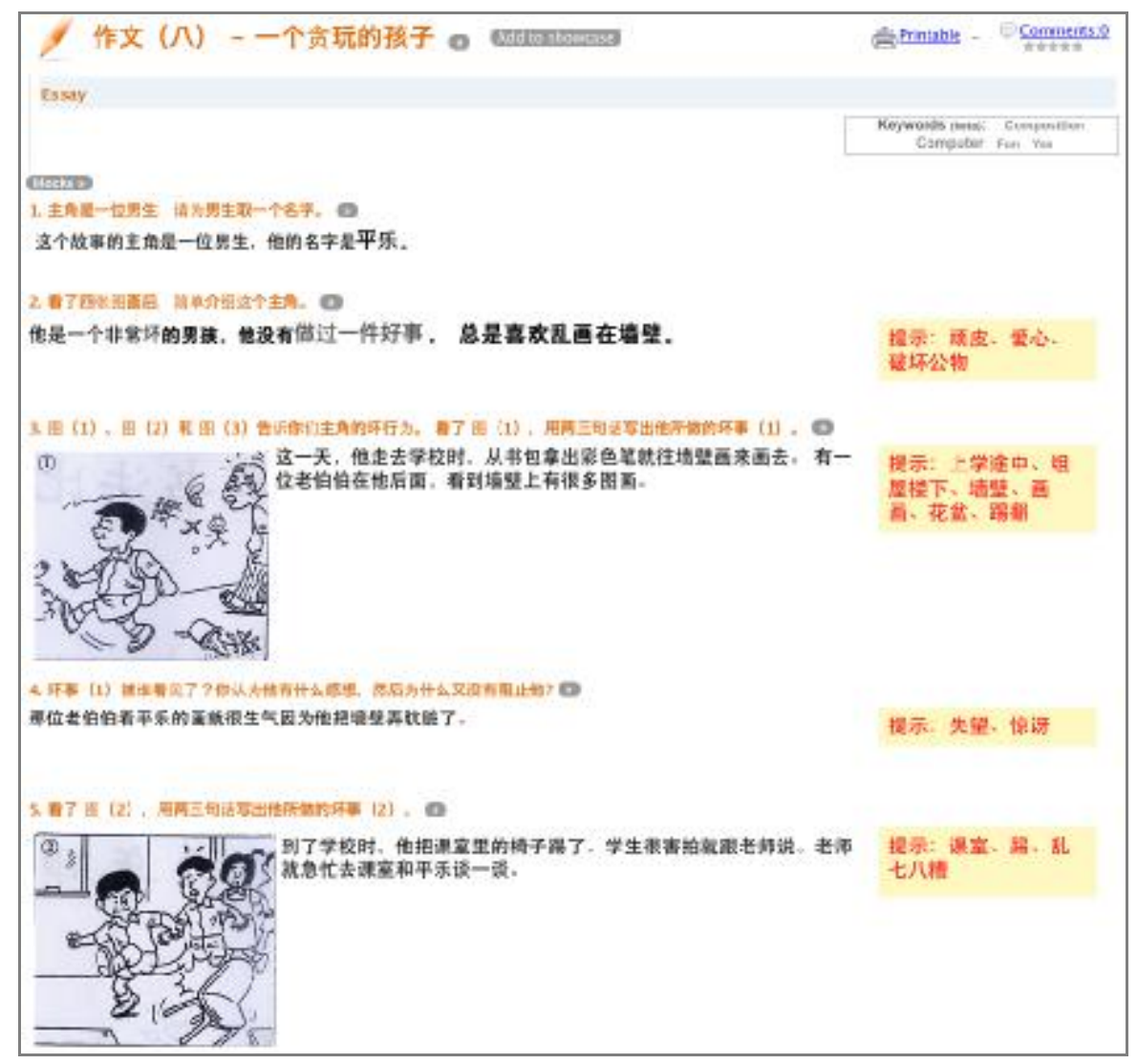

Figure 2. A scaffold with question prompts and helping words (text on the right).

\subsection{Findings from the Pilot Study}

The pilot study showed that students enjoyed learning in an ICT-mediated environment. The teacher indicated that many students previously disliked writing compositions but since using ICT to complete compositions, they would look forward to composition lessons. In addition, students were more motivated to do their work properly especially for ICTmediated assignments. It also appeared that behavior in the class improved as students realized that when they behaved well, the teacher brought them to the computer-lab (Personal email communication, March 21, 2009). In the post-pilot student survey, eighty percent of the students indicated that they valued the ICT-mediated writing environment. They said that it increased their confidence in writing as the ICT platform provided them with writing prompts and additional 
helping words. More importantly, the positive effects as a result of using ICT were not novelty effects as the advantages sustained over the entire year. This may have been due to the engaging, immediate feedback nature of ICT since students, for example, were especially excited about a Chinese Language game that helped them improve their pinyin (i.e., phonetic skills) and built up basic language skills.

On the other hand, the pilot also clearly showed that students faced common challenges in writing Chinese:

1. Most students had limited vocabulary. For example, they may only have one word for a description and they used it for all situations, e.g., they use simple descriptions such as happy (高兴, 开心) instead of more vivid or precise descriptions such as excited or high-spirited (兴奋，兴高采烈).

2. Students had problems with sentence construction. All except one student needed training to improve their sentence structure. The lone exception was a native speaker of Chinese originally from China. The teacher indicated that students could not write grammatically correct sentences. One common problem was that they would translate sentences literally from English such as 我要吃饭在这里 (I want to eat here) instead of the grammatically correct sentence 我要在这里吃饭 (I want here eat).

3. Students' writing did not flow. Students had problems linking sentences to describe an event, although some were able to verbalize it. This may indicate cognitive overload in translating from verbal to written form. Furthermore, students did not know how to make use of linking words such as meanwhile and after. So they wrote in short, often disjointed, sentences. This caused the breakdown of flow in the composition.

4. The compositions were plain and had only basic short descriptions of the four pictures provided to guide students in their writing. The compositions lacked feelings, description of events, and character portrayal. It was clear that students did not have adequate vocabulary and they lacked composition-writing skills. For the weaker students, writing compositions simply meant completing the number of characters (80) required. It was observed that weaker students would frequently count the number of characters in their compositions.

From the pilot, it was clear that a writing program was needed to help students improve their basic writing skills such as transcribing, use of appropriate and interesting vocabulary, and how to write flowing sentences. At a higher level, they had to be taught composition writing skills to improve the vividness of their writing and opportunities for collaboration and peer/teacher feedback needed to be provided.

This paper focuses on the latter effort, where the study of one writing skill, that of the "action chain", was conducted as part of normal curriculum based teaching in a primary 4 class in a neighbourhood school. The objective was to study whether an ICT-mediated writing program guided by the scaffolding research would improve writing quality and pedagogical engagement in a real world school environment.

\section{The writing program}

The writing program aims at providing effective differentiated learning by incorporating past research findings as well as addressing issues the school faced.

\subsection{Incorporating prior research findings on scaffolding}

In 1976, Wood, Bruner and Ross adopted the term "scaffolding" to describe a social interactional structure present in the environment that guides the student to achieve learning outcomes. These scaffolds often take the form of leading or probing questions that is especially conducive to helping children learn (Foley, 1994). Scaffolding is gradually removed as the learner increasingly gains mastery of the learning task.

Today, the concept of scaffolding adopted in the classroom is known as instructional scaffolding. Here, the metaphor is extended to address what, when, and how to scaffold and when to fade scaffolding (Lajoie, 2005). Davis and Miyake (2004) highlighted a range of issues such as: the teacher needs to perform just-in-time assessments for each learner in the classroom and to provide calibrated support based on the assessments. This is rarely achieved in practice in a real classroom situation though calibrated support can be partially offloaded from the teacher through adopting computer technologies. The limitation is that while computers work in real time, the systems do not accurately diagnose the abilities or performance standards of the learners to provide accurate supports or fading of scaffolds (Davis \& Miyake, 2004).

Furthermore, in a classroom, the teacher need not be the only means of providing scaffolds to students. Other means include computers, peers, and online experts. It is important to consider how the scaffolding responsibilities are distributed among such means. From this perspective, Tabak (2004) highlighted three types of scaffolding, namely, differentiated scaffolds, redundant scaffolds, and synergistic scaffolds. Differentiated scaffolds are devised and each scaffold supports a different need. Redundant scaffolds basically provide multiple scaffolds to support the same need. This is to help students who have different learning needs with respect to a learning goal, e.g., if the student cannot benefit from one scaffold, he or she can rely on the support of another type of scaffold to achieve the learning goal. Synergistic scaffolds are different scaffolds that complement one another to help students achieve a goal. For example, software scaffolds alone may not be able to explain the rationale behind certain procedures or strategies. The teacher has to instruct students before they use the software scaffold and also, the teacher needs to provide dynamic reasoning 
while students are using the scaffold. Tabak further pointed out that it was important that the teacher integrated the scaffolds into a coherent system of learning.

Differentiated fading in the classroom is seldom addressed in research and very little empirical reports have been made on the issue (Pea, 2004). Pea pointed out that a fading process needed to be implemented to lead learners to independent performance. Such proof must be furnished for each learner, not only for selected groups or learners.

\subsection{The school setting}

We learned from the pilot findings that while students were very enthusiastic in learning to write Chinese compositions via ICT, the teachers were severely challenged by the limited lesson time allocated to teach Chinese compositions in big classes of mixed-ability students. This is especially difficult when many students were weak in their basic language skills and composition writing skills.

Due to the large class size (typically greater than 30 students) and the time constraints mentioned, the instruction and help that teachers can provide could only be one-size fit all. Due to severe time constraints, teachers did not have the time to individually evaluate student performance and provide customized just-in-time help. Thus, differentiated teacher support for students was lacking.

Moreover, the instruction and helping words provided were often specific to each composition. They were faded at the end of the lesson. As composition instruction focused on writing the given stories and words specific to the stories, they could not be repeated in the next composition lessons. High-ability students were able to recall and use these words in appropriate situations but lower ability students could not. This may be because the lower ability students had exceeded their zones of proximal development. Differentiated fading would allow higher ability students to learn beyond what was given and lower ability students to learn within their abilities.

The writing program addressed the issues the school faced by the following methods:

\subsubsection{Introduced micro-writing skills}

Scaffolding was the primary building block of the program. As such, we address the one-size fit all blanket help provided in class by creating small chunks of writing strategies (i.e., instructional scaffolds) that students could learn in a short period of time and which could be applied in a wide variety of contexts. They are also easy for teachers to scaffold students throughout the academic year. Thus, we designed and taught writing strategies such as the Big Four ("when", "who", "where", and "what") for creating good introductions, Feeling Chains which helped students add adverbs to descriptions, and Action Chains which taught students how to describe an event more vividly by breaking it down into a sequence of three closely related actions. These strategies could be reinforced in composition lessons throughout the year thus allowing students to internalize them at their own pace. In practical terms, these scaffolds need not be faded at the end of just a single lesson.

\subsubsection{Adopted ICT-mediated scaffolds to guide Chinese composition writing}

In response to Tabak's (2004) call for integrating differentiated, redundant, and synergistic scaffolds to create a coherent learning system, three types of writing scaffolds were integrated into the writing programs. First, to address the needs of higher ability students, differentiated scaffolds were created by combining writing strategies. For example, after higher ability students learn the basic strategy for writing action chain, they could proceed to combine character portrayal with an action chain, e.g., the old man with grey hair, fading eyesight, walked wobbly with a cane (underline indicates character portrayal), stepped on the rock, lost his balance and fell heavily on the ground with a big "thud" (italic text indicates the action chain).

Besides the main scaffold, a series of redundant scaffolds such as electronic worksheets and a collaborative mindmap to brainstorm for action words were used to help lower ability students learn writing strategies.

In the main scaffold for action chain, students were grouped up to work on assigned actions online. There were three nodes for them to collaboratively come up with three closely related actions. Once a group member updated a node, it would show up on the group members' computer screen (see Figure 3). Finally, students had to write a sentence each that includes the three closely related actions. In addition, the teacher provided two additional variations of action chain template on paper for students to fill in individually. When writing compositions, she also provided helping words that students could use to write action chains.

To scaffold synergistically, the teacher conducted direct teaching, did modeling, and adopted the ICT-mediated collaborative mind map. These learning activities complement one another to help students achieve the goal of writing vivid actions. In direct teaching, the teacher first introduced the goal of writing action chain. Then, she showed video clips of actions and asked students to help break them down into three closely related actions. She also showed examples of well-written action chains. Finally, she modeled how to fill out the three boxes in the template. After she has completed direct teaching and modeling, she let students work on the ICT-mediated scaffold online. 


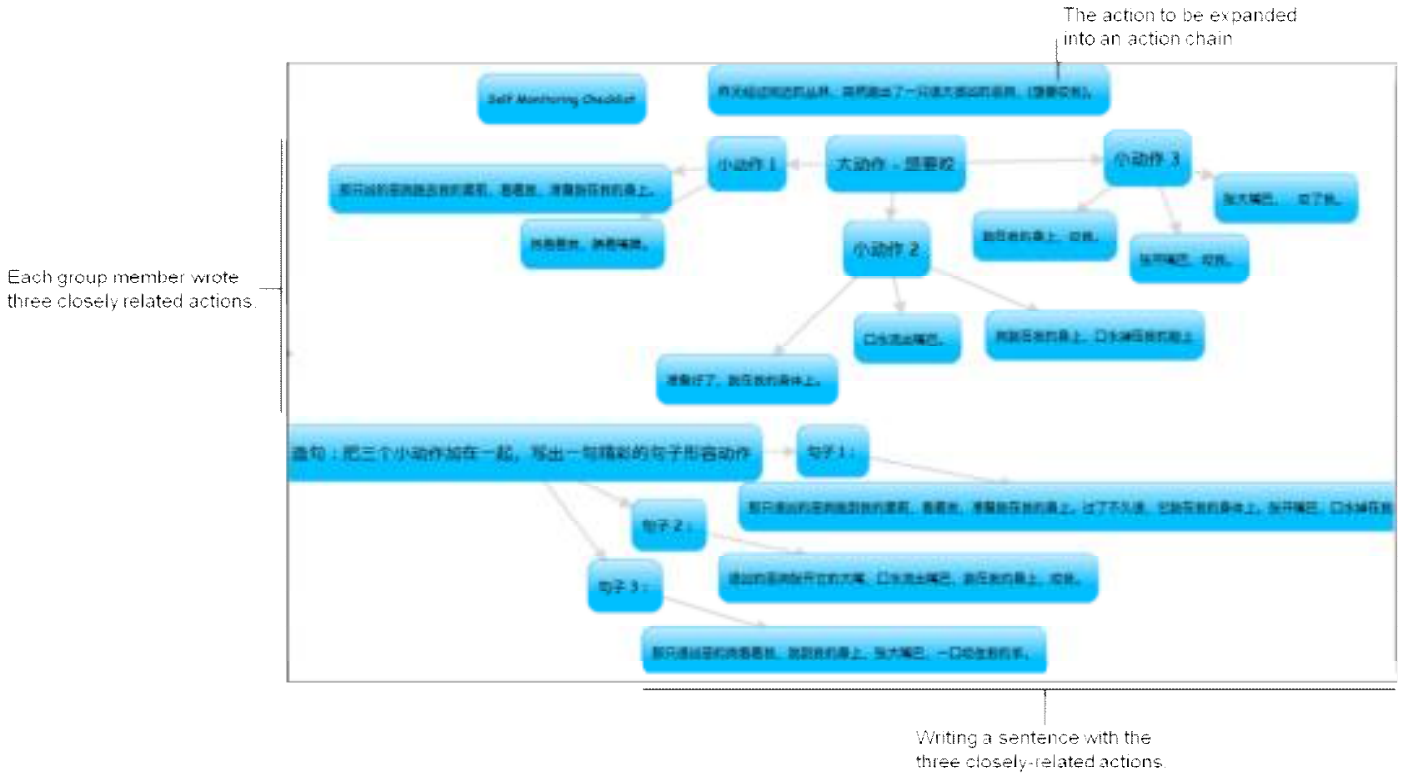

Figure 3. A collaborative mind map scaffold to help students write action chains.

\subsubsection{Engaged peers as scaffolders}

With ICT, the teacher could provide more differentiated support to address the needs of students. The mind mapping application allowed teachers to create a simulated environment in which students could immerse themselves and collaborate in Chinese. This could offload at least the transcribing responsibilities from teachers, which normally would take up a large chunk of the composition lesson time, to students. The teacher then had more time to work with students who needed her more.

The writing program adopted ICT-mediated scaffolding as its building block. Its aim was to help students apply microwriting strategies so that they could write vividly. These strategies are small for ease of learning and applying. In this paper, we will focus on the action chain micro-writing strategy. The main scaffold for writing action chain was the ICTmediated collaborative mind map in which students collaboratively came up with three closely related action, one in each node, then they each write a sentence that consisted of the three related actions. The ICT platform provided the environment for collaborative brainstorming and writing composition with scaffolds. Students' works were recorded for analysis and assessment.

\subsection{Research Questions}

This study attempted to answer these questions:

1. What is the best way to integrate ICT-mediated scaffolds effectively to guide Chinese composition writing?

2. How do ICT-mediated scaffolds increase differentiated teacher support in class?

3. How can fading be implemented effectively?

\section{Methodology}

A mixed qualitative and quantitative case study design was adopted for this study. This design allow us to experience and document the teaching of Chinese composition writing in a local primary school under real world conditions.

\subsection{Data Collected}

Various types of data were collected and analyzed. They were:

\subsubsection{Students' artifacts}

There were two kinds of student artifacts - work done on paper and work done online in the e-portfolio. Artifacts available for analysis that were done on paper included the pre-test compositions of the whole class, scaffolding exercises and compositions written online.

\subsubsection{Teacher's observation notes}

The teacher took detailed observation notes on the implementation of the various writing strategies and learning activities (e.g., ICT-mediated activities, writing in the e-portfolio). Areas the teacher took notes on included students' performance, the difficulties she or students encountered in class, effectiveness of the ICT-mediated writing strategies, effectiveness of group work, and the role of the teacher in an ICT-mediated classroom. The teacher's observation notes were written in a blog in her e-portfolio. It is password-protected. 


\subsubsection{Personal communication with the teacher}

This was conducted via email whenever we needed to clarify issues with her, e.g., how did some of the students perform in other subjects, her perception of the effectiveness of writing strategies, how did she conduct certain writing activities?

\subsubsection{Students' surveys}

A total of 47 students took a survey at the end of the study. The survey was administered online in the computer lab at the end of the year. The survey was created using Survey Monkey. It assessed students' perception on the effectiveness of the writing strategies. Responses were analyzed as a whole as well as grouped into high-, medium-, and low-ability in Chinese categories. There were 18 high-ability students, 15, medium-ability, and 20 low-ability students.

\subsubsection{Students' interviews}

At the end of the study, ten students (two high-ability, six medium-ability, and two low-ability) were interviewed by the teacher. They were interviewed to find out more about their perception of the writing instruction intervention. The interviews were semi-structured. The teacher would ask students an open-ended question. If they could not answer, she would prompt them with options. They were asked in-depth questions (e.g., why were certain writing strategies useful or not useful for them, what specifically did they feel they needed to improve in).

As the mixed quantitative and qualitative approach was adopted to analyze the data collected, statistical analysis was conducted on composition scores. Students' artifacts, teacher's notes and students' responses in surveys and interviews were analyzed qualitatively. The aim was to find out if different sources of evidence corroborate one another. If they did, there would be stronger support for the writing program.

\subsection{Grouping students into high-, medium-and low-Chinese ability for data analysis}

To attain greater granularity of results, the performance of different ability students was analyzed. Student clusters were categorized according to their exam scores in curriculum material in the Continual Assessments (CA) 1 and 2 which took place in March and September. These assessments included comprehension of text, learning of vocabulary, and sentence structure. These two exams did not have a composition component. As such, the grouping was based on the student's general Chinese language ability independent of their writing skills. The mean CA scores, which ranged from 11 to 48, were used to categorize students into high, medium, and low ability. Natural boundaries were identified at clear gaps in the average scores. One gap occurred between 41.5 and 40 which was set as the boundary between high and medium ability students; another occurred between 35.25 and 30.5 , which was set as the boundary between medium and low ability students.

\section{Results and Discussion}

\subsection{Teacher as scaffolder}

The teacher scaffolded the action chain micro-writing skill to help students write more vivid actions. She first showed students how she expanded an action into three closely related actions. Then she chose an action such as "ride a bike" for students to brainstorm for closely related actions. According to the teacher: "The brainstorming activity is conducted as a whole class where better ability pupils provide their observations of certain actions and teacher assists in recording the actions into words" (April 7, 2009). The teacher then drew three boxes and guided the students to fill in boxes with one closely related action each. Then, she asked each student to write a sentence with the three closely related actions the class had come up with. The final sentence writing activity was an important step to tell students it was not enough to break down an action into three actions. They had to write a sentence with the three closely related actions, which was the goal of the exercise.

\subsection{ICT-mediated scaffold via collaborative mind maps}

The teacher also scaffolded students to expand actions into related actions via the ICT-mediated collaborative mind map. When scaffolding students via the collaborative mind map, they were grouped up and each group was given a phrase to expand into related actions. Each group had their own mind map and every member in the group could edit it on their own computer screen and they could edit one another's work. The teacher commented how she grouped students:

For this activity the pupils are grouped into small groups of 3 or 4 . Each group works on one mind map. In their group, there is a mixture of weak and strong pupils. This is to enable the strong pupils to guide the weaker ones during discussion, allowing the weaker pupils to model and learn from them (Teacher's note, April 18, 2009).

\subsection{The teacher's comment on the effectiveness of working collaboratively via the mind map.}

The teacher found that students would gradually adapt to working with peers closely online. The software was able to:

1. Improve student engagement. The teacher noted that "pupils are excited about the activity. Though it's their first time using the mind mapping function, most of them are patient and are willing to try out the functions" (April 18, 2009). She further noted that: 
... they take longer time to complete the assignment, however, I can see that the pupils are more interested in ICT based than pen-paper based. Pen-paper though discussion is face-to-face, weaker pupils may not open their mouth to contribute. But by this new method of pulling individual nodes, the weak pupils are forced to contribute their own nodes. Even if they are just copying from the stronger team mates, at least that action is learnt and absorbed. (this is modeling) (April 25, 2009).

2. Improve student collaboration skills. After students had more practiced using the mind map software, they improved in collaborative work. They would collaborate verbally and then make changes online. This is reflected by the teacher's comments after the second mind map activities:

We realised this time, there are verbal communication between stronger pupils. They are sharing ideas how they can improve on the sentence. Communication is verbal which is easier for them, instead of expressing what they want to say through typing in the comments column (April 25, 2009).

In addition, ICT enabled students to collaborate from different locations:

... using ICT allows children to work on the assignment anywhere. One kid was unwell and stayed at home. However, during the ICT lesson, he logged onto the website and was able to communicate/discuss with his group mates and complete the assignment with them (April 18, 2009).

3. Encouraged students to learn independently. The collaborative mind map empowered students to be independent in learning. The teacher noted: "I allow the better pupils to have the freedom of completing the activity independently, only to show me the outcome of the assignment before shutting down" (April 18, 2009). It also enabled the teacher to teach at a higher, or, conceptual level: "...I only help them through simple verbal prompts like you can think of how to expand this action etc." (April 25, 2009). The teacher summarized her teaching experience via the collaborative mind map as follows:

We made use of the mindmap to let students accumulate descriptive vocabulary and also work on action chain. Because their peers' work are reflected real-time on their screens, the H[igh-ability] students are able to learn independently, by reading other peers' work then reflect and amend their own sentences. I am able to then spend more time on the W[eak] students, helping them on the action chain sentence making (July 2, 2011).

\subsection{The teacher's comments on the effectiveness of the action chain micro-writing skill.}

The teacher perceived the action chain intervention to be the most difficult writing skill to scaffold. It was because students had to visualize the unfolding of an action, which was something they had never learnt to do before. Furthermore, they had to describe the related actions with words that they most likely did not have in their vocabulary. Furthermore, there was not enough time dedicated in the teaching of the action chain writing strategy: "Due to time constraint, I was not able to get pupils to act out the actions. I only got the pupils to think and give me suggestions of small actions" (April 7, 2009).

Contrary to her belief, students performed surprisingly well in applying the action chains writing skill. The teacher was also amazed by her students after she introduced action chains to them for the first time: "Some of the pupils have already included simple action chains in their composition" and "the weaker pupils actually [were] able to tell you the small actions but their usage of words limited, hence teacher needs to provide more prompts on this" (April 7, 2009). The teacher also noted that "all the pupils are on task and are able to come up with 3 tiny actions to support the big action. They are also able to link the tiny actions into a sentence, forming an action chain" (April 18, 2009).

\subsection{Students' comments on the effectiveness of the action chain micro-writing skill}

Those high- and medium-ability students who could write action chains showed confidence in their abilities: "I am able to split the action into smaller actions"; "[They] make composition more interesting plus get more marks"; "get to know many action vocabulary (more exposure to them)"; "Teachers' marks also reflect I do well in these"; "I can imagine the actions; and I have the vocabulary to write action chains". On the other hand, many students commented: "very hard, I was not able to write them. It is too difficult. Too difficult and always get it wrong”.

What is it then that caused everyone to perceive action chain as a difficult writing skill to master? Some students reported:

(a) They were not able to visualize the unfolding of an action and as such, they were not able to write the three closely related actions. A student commented: "I can only think of two actions". Other comments included:

I can't really see it in my mind's eye so I don't have anything to write about. I know where to add action chain but am unable to write as specifically and I am not able to break down [an action] to tiny actions.

(b) Students did not have the vocabulary to write detailed actions, as a high-ability student commented, "I need more vocabulary";

(c) They did not know where to add action chains, that is, they could not identify actions to write in the pictures: "I don't know where in the pictures to add actions". 
Furthermore, students commented that there was not enough time dedicated to the teaching and learning of action chain activities. A high-ability student commented: “... too little time spent, a little rush as we cannot discuss/elaborate much in class" and "... too few actions are discussed". There was also "too short a time to absorb learning".

Students found it difficult to write action chains. Did they actually write them in their compositions? Let us take a look at the compositions in the e-portfolio to find out how they performed in writing action chains.

\subsection{Action chain performance in the compositions written in the e-portfolios.}

Students did well in applying the action chain writing skill to describe actions. In Composition 5, even the low-ability students could break down an action into at least four closely related tiny actions. For example, JN, a low-ability student, wrote (italic text indicates closely related actions): "This day, When Grandpa Wang was sweeping the floor, he accidentally knocked on the metal cabinet. The very precious vase was toppled. 'Clang' that vase fell on the floor". Another low-ability student TT described the incident in this manner: This day, Grandpa Wang reached the office. He went to sweep floor at once. When he swept till the boss' office, grandpa Wang swept till he reached the boss' cabinet. He stepped back, the vase dropped, and broke. BL, another low-ability student, wrote a six-component action chain: This day, old grandpa as usual, was sweeping at the boss' office. Accidentally stepped back, his buttock knocked into the metal cabinet, the boss' favorite vase was knocked over and broken. The glass shattered on the floor.

High- and medium-ability students could write better quality action chains. For example, JC could write action chains with logical flow and imagination (italic text indicates closely related actions):

This day, Uncle Lin was sweeping the floor in a corner of the office. He did not notice that behind him was a cabinet. On top of the cabinet was a vase, that vase was the boss' beloved vase. Uncle Lin accidentally knocked over the vase. The vase dropped and shattered with a "clang".

A medium-ability student AQ also wrote with better basic language skills than the low-ability students (underlining added to indicate closely related actions):

That day, Uncle Wang, as usual, went to the office at 7 o'clock to clean up. Uncle Want went to the boss' office to sweep the floor. When he swept, backed up a step, the back knocked into the cabinet accidentally, the vase on top of the cabinet dropped, the vase shattered with a "clang".

High-ability students were able to apply the action chain writing skill to describe actions in detail and with imagination while medium-ability students' descriptions were effective and in 'good' Chinese and with some imagination. Although low-ability students were able to breakdown an action into a few closely related actions and described them in 'simple' Chinese, they missed including relevant events. As a result their description did not make sense in some places.

Since action chain was perceived to be difficult to write, why was it that students were able to write good action chains in Composition 5? It could be because the teacher anticipated the challenges students faced and helped them to resolve the issues by first, letting students practiced writing action chain before the actual composition. The teacher explained:

We made use of Silverlight mind mapping to do brainstorm for tiny actions for some big actions like fighting, falling etc. With the tiny actions, they will put them together and form sentences (May 2, 2011).

As students worked on writing action chains via collaborative mind maps just before this composition, the writing skill was therefore fresh in their mind and they were able to render it during the composition.

The teacher included appropriate vocabulary for writing the action chain as helping words. They were words like sweeping the floor, metal cabinet, favorite vase, backing up, and accidentally. By using these words to form sentences, students could write an action chain.

Hints were given and the teacher inserted an instructional prompt at the appropriate textbox to tell students where to write the action chain. In addition, the action was indicated in the picture. Students knew exactly where to insert the action chain.

Did students internalize the writing of action chain? Let us take a look at whether students wrote action chains in Composition 9, which was the last composition written for that year. In the pictures and the e-portfolio, there were no hints given to students as to where to write an action chain. If students could write action chains in this composition, then it would provide support for the claim that they had internalized the writing of action chains.

In this composition, there were no actions in the pictures. However, students could still include a sequence of events (event chain) in their compositions. The teacher would regard this as a natural extension of writing action chains as event chains could not be clearly separated from action chains in the first place. In fact, in action chain writing exercises, the teacher did not differentiate between the two. For example, students could write an action chain or an event chain for the phrase picking up a wallet from the floor. The action chain version could be: She spotted the wallet on the floor. She looked left and right several times, walked towards it, bent down and picked up the wallet quickly. An event chain could be: She spotted the wallet. She went over to pick it up from the floor. Then she made her way to the police station to hand it over to the police. The teacher would award both with higher marks for vividness.

Did students write event chains in the final composition? Low-ability student TT described what Ming, the character in the story, did when he got home (italic text indicates components in the event chain): "This day, after school, Ming 
reached home, cast his schoolbag aside, then he switched on the TV to watch cartoon". Another low-ability students wrote $\mathrm{JN}$ a combination of event chain and action chain: "This day, after school, Ming, once reached home, went to shower and eat lunch immediately. After lunch, he dashed to watch TV program. He switched on the TV, unable to take his eyes off the TV program".

$\mathrm{AQ}$, a medium-ability student, wrote this event chain (underlining indicates components of the event chain): "This day, after school Ming, upon reaching home, cast his school bag aside, turned on the TV, with eyes wide open, began to watch TV programs".

TXH, a high-ability student, wrote a long event chain: "This day, after school, Ming got back from school. Once he reached home, he immediate put his school bag down, switched on the TV then sat on the sofa to watch TV and be enthralled by the program".

Composition 9 has provided us with evidence that students had acquired skills of writing closely related actions or events. We can see sequences of events in compositions of different ability students. Although it was originally thought that this was the most difficult micro-writing skill for students to learn, it turned out that they could acquire it in a short time.

Students were able to apply the action chain micro-writing skill. However, medium- and low-ability students would need scaffolding to help them better describe closely related actions. They lacked the vocabulary or sentence structure to write effectively.

Although the action chain micro-writing skill can be learned quickly, the teacher may have to provide regular opportunities for students to write action chains. This will reinforce them to automatically identify places where they could add an action chain, visualize how an action unfolds, and use appropriate vocabulary. The weaker students must learn more actions words. This can be acquired through Chinese language games. The following statistical analysis of students' performance in writing action chains showed that students of all ability could write action chains, contrary to the teacher's belief.

\subsection{Statistical analysis for action chains}

To find out if students wrote more action chains over time, the number of action chains and event chains in the pre-test, Compositions 5 and 9 were compared. In Composition 5, there was a distinct action depicted in the one of the pictures. The action could be broken down to a series of tiny related actions. The teacher also instructed students where to include an action chain. In Composition 9, there was no such depiction in the pictures and the teacher also did not instruct students to write an action chain. As the teacher also taught her students to expand an event into closely related events, the concept of action chain could be expanded to include writing a sequence of events.

A repeated measure ANOVA was conducted to compare the numbers of action/event chains written in the three compositions. Sphericity was tested using the Mauchly's Test, which finds out if the hypothesized and the observed variance patterns were equivalent. The test was significant, $W=.65, \chi^{2}(2)=8.96, p=.01$, which indicated that the assumption of sphericity had been violated. Therefore, degrees of freedom would have to be corrected. In this case, the epsilon values from Mauchly's test values are .74 and .78 , both very close to .75 . As such, the Huynh-Feldt estimate of sphericity corrected value $(\varepsilon=.78)$ is used.

It follows that the number of action/event chains for the three compositions differed significantly, $F(1.57,34.47)=$ $39.74, p=.001 . \eta_{\mathrm{p}}{ }^{2}=.64$ (see Table 1). Post hoc comparisons show that students wrote significantly more action chains in Compositions 5 and 9, compared to the pre-test. The results supported the findings from the analysis of the compositions that students were able to write action chains.

Table 1. Descriptive Statistics for the Number of Action Chains in Three Compositions and P Values for Pairwise Comparisons

\begin{tabular}{lcccc}
\hline Composition & $\mathrm{M}$ & $\mathrm{SE}$ & Pre-test & Composition 5 \\
\hline Pre-test & .00 & .00 & & \\
\hline Composition 5 & 1.00 & .09 & $.001^{*}$ & \\
\hline Composition 9 & 1.13 & .16 & $.001^{*}$ & .42 \\
\hline
\end{tabular}

Note. $\mathrm{N}=23 . \alpha=.05, *=$ significant difference

5.7.1 Differences in number of action chains written by high-, medium-, and low-ability students.

Did all students benefit from the action chain writing strategy and write more action chains? We conducted a one-way between groups ANOVA to find out. The total number of action chains written by each student was first computed. Test of homogeneity of variance was not violated $(p=.06)$.

Results showed that there was no significant differences in the number of action chains written by different ability students, $F(2,22)=1.19, p=.33, \eta_{\mathrm{p}}{ }^{2}=.11$ (see Table 2). Results suggested that students of all ability levels benefitted from the action chain scaffolding. 
Table 2. Descriptive Statistics for the Total Number of Actions Chains Written by Different Ability Students and P Values for Pairwise Comparisons

\begin{tabular}{lllcc}
\hline Composition & $\mathrm{M}$ & $\mathrm{SE}$ & High & Medium \\
\hline High & 2.42 & .36 & & \\
Medium & 1.75 & .16 & .30 & \\
Low & 2.00 & .00 & .78 & .92 \\
\hline
\end{tabular}

Note. $\mathrm{N}=23 . \alpha=.05, *=$ significant difference

It is therefore safe to conclude that the action chain scaffolding in the writing program was successful in helping all levels of students describe actions vividly. All students wrote more action chains after the scaffolding and some students broke down actions into more than 3 closely related actions. Statistical analysis was required to find out if higher-ability students break down an action into more than three closely related actions. The total number of closely related actions was first computed for each student. Then the data were analyzed using the one-way between groups ANOVA procedure. The assumption of homogeneity of variance was not violated $(p=.07)$.

Results suggested there was no difference in the number of closely related actions written over time by different ability students were $F(2,22)=2.16, p=.14, \eta_{\mathrm{p}}{ }^{2}=.18$ (see Table 3$)$.

Table 3. Descriptive Statistics for the Total Number of Closely Related Actions Written by Different Ability Students and P Values for Pairwise Comparisons

\begin{tabular}{lllcc}
\hline Composition & $\mathrm{M}$ & $\mathrm{SE}$ & High & Medium \\
\hline High & 9.83 & 4.80 & & \\
Medium & 6.25 & 2.12 & .12 & \\
Low & 8.00 & 1.00 & .74 & .78 \\
\hline Note. $\mathrm{N}=23 . \alpha=.05, *$ significant difference
\end{tabular}

Analyses indicated that the action chain micro-writing skill was, contrary to our belief, not a difficult concept for students to learn and apply. From the teacher's observation, it seems that low-ability students did not have much of a choice but to write their action chains in awkward Chinese. Medium-ability students were precisely the group that would spend time trying to write better Chinese. As such, their mental effort and attention were directed at improving their basic language (e.g., write better sentences, use more vocabulary) rather than writing actions chains. In future interventions, it may be beneficial for the teacher to introduce video clips in the scaffold so that students can select appropriate frames to describe. This will help medium-ability students better focus on describing actions. It will also help high-ability students to write more enriched action chains. However, for the weaker students, improving in their basic writing skills is still the key to better writing performance.

\subsection{What students Enjoyed Most in the Action Chain Learning Process?}

When the teacher asked what students enjoyed most in learning to write composition, all high- and medium-ability students (altogether eight students interviewed) selected group work as the activity they like most. They said they "enjoyed working in groups", "like my friend to edit my work", and "interesting to learn with friends". They also requested to "have more group work on sharing among friends" as it would enable them to "learn some good ideas/writing techniques", "discuss on what to write", "discuss on new vocabulary", "play [act] out the pictures for understanding of them". In future interventions, ICT-mediated group activities should again be integrated to scaffold students. Low-ability students did not find group work interesting probably because they did not contribute much to the group activities. It seemed that students selected the activity they could do well in as the activity they liked best.

\subsection{Suggestions and future plan for improving the writing program}

Besides setting aside more time to teach and for students to practice writing action chains, we should also let them explore how actions unfold. This will improve their visualization skills. For example, a high-ability student made the suggestion of using video to help students visualize unfolding of actions:

Video will be able to help me, and [there was] little time spent/taught this in school so when reading at home I will go and find out more on action chain which make me more interested in this part; mom will guide me/teach me to write in details (through thinking).

Video is an ideal tool to show how actions unfold. Using video clips to teach the writing of action chains, the teacher can pause at the appropriate frames and ask students to write descriptions about them. 
In addition, the teacher provided the suggestion to introduce good action chains writing samples from previous classes so that students know what good action chains are like. The teacher commented: "The example from the pupils' composition became an introduction to the lesson. I explained to the pupils what action chain is and that for vivid actions, they should observe more and break the actions down into smaller actions" (April 7, 2009).

In the following paragraphs, we will discuss work in the progress to integrate video functionalities and create a database for action chain samples into the software platform. It is hoped that with these functionalities, the writing program can further help students acquire and internalize the action chain micro-writing skills.

To help students visualize and describe closely-related actions, an iPad application has been developed for students to capture an action clip. They can then view each frame in the video clip and select the frames they want to add descriptions. The video clip, together with the descriptions, will be uploaded to a dedicated work-area in the software platform for teachers and peers to comment on. The teacher can then link the video clip to individual students' eportfolio for them to make further refinements.

Students' video action chain clips annotations and their compositions are valuable resources to scaffold subsequent batches of students. They can help students grasp what the teacher means by writing good action chains. In the short term, we are working towards including functionalities to help teachers identify good work and make them available for demonstration. In the long term, the system will mine artefacts and automatically retrieve appropriate resources to scaffold students toward writing better action chains (Chung, Leong \& Loo, 2006). Such scaffolds are known as adaptive scaffolding (Pea, 2004).

\section{Conclusion}

The teacher integrated a suite of ICT-mediated scaffolds to address the different needs of students. She incorporated differentiated scaffolds to ensure students of various abilities could work within their own ZPD (Zone of Proximal Development). Weaker students worked on simpler task while higher ability students had more complicated tasks to challenge them. She also created redundant scaffolds to provide additional practice for very weak students. Her scaffolds complemented one another to create a coherent learning system. Furthermore, she scaffolded synergistically by conducting direct-teaching, demonstrating good and bad examples, modeling, and allowing students to work on ICTmediated activities. Analysis of students' compositions, the teacher's and students' feedback indicated the scaffolding activities improved students' performance in writing vivid actions. All students were able to construct action chains. And different ability students did not differ in the number of closely-related actions they wrote. Students also wrote significant more action chains in their compositions compared to the pre-test.

With ICT, the teacher could conduct peer-editing activities with relative ease. Working collaboratively online, students also received just-in-time feedback from peers, which motivated them to persist with the task at hand. The teacher was freed from providing routine help for the average students, which usually took up the bulk of her instruction time in the traditional setting. She then had more time to help the high-ability or very weak students.

With the introduction of small chunks of writing strategies, students could learn them quickly and apply them to the compositions they wrote. The teacher could scaffold them throughout the year and fade them only when the students had mastered their work. With micro-writing strategies, teachers are able to extend the practice time, which is critical in consolidating learning.

After the pilot study, the school started grouping similar ability students in a class for ease of teaching and scaffolding. They also incorporated the various micro-writing strategies and scaffolds in their composition lesson plans. Students can therefore benefit from a coherent learning program, which enabled them greater flexibility to learn at their own pace.

\section{References}

Chung, T., Leong, M., \& Loo, P. (2006). Automated mentoring for reflection in an e-portfolio. Proceedings of the 14th International Conference on Computers in Education. In R. Mizoguchi, P. Dillenbourg, \& P. Zhu (Eds). Learning by effective utilization of technologies: Facilitating intercultural understanding, (pp. 457-464). Amsterdam, Netherlands: IOS Press.

Davis, E., \& Miyake, N. (2004). Explorations of scaffolding in complex classroom systems. The Journal of the Learning Sciences, 13(3), 265-272.

Dixon, Q. L. (2005). The bilingual English policy in Singapore: Implications for second language acquisition. In J. cohen, K. T. McAlister, K. Rolstad, \& J. MacSwan (Eds.). Proceedings of the $4^{\text {th }}$ International Symposium on Bilingualism (pp. 625-635). Somerville, MA: Cascadilla Press.

Foley, J. (1994). Scaffolding, ELT journal, 48 (1): 101-102.doi: 10.1093/elt/48.1.101 Retrieved 24 February, 2014 from: http://eltj.oxfordjournals.org/content/48/1/101.short 
Lu, J., Zhang, C., \& Qian, P. (2002). Characteristics of the grammar structure of the Singaporean Chinese language. In C. H. Chew (Ed.). Vocabulary and grammar structure of Singaporean Chinese language (pp. 75-148). Singapore: Lingzi Media.

Lajoie, S. (2005). Extending the scaffolding metaphor. Instructional Science, 33, 541-557. Retreived July 15, 2008, from SpringerLink Contemporary database.

Ministry of Education (MOE) Singapore (2012). Education in Singapore, MOE, Singapore. Retrieved 24 February, 2014, from: http://www.moe.gov.sg/about/files/moe-corporate-brochure.pdf

Moyer, A. (2004). Accounting for context and experience in German (L2) language acquisition: A critical review of the research. Journal of Multilingual and Multicultural Development, 25(1), 41-61. doi: 10.1080/01434630408666519

Pakir, A. (2008). Bilingual education in Singapore. In J. Cummins and N. H. Hornberger (Eds.), Encyclopedia of language and education, $2^{\text {nd }} \mathrm{Ed}$, Vol. 5: Bilingual Education, pp. 191-203.

Pea, R. (2004). The social and technological dimensions of scaffolding and related theoretical concepts for learning, education, and human activity. The Journal of the Learning Sciences, 13(3), 423-451.

Sim, S. H. (2005). Teaching Chinese compositions in Singapore secondary school. In M. S. Shum, \& D. L. Zhang (Eds.). Teaching writing in Chinese speaking areas (pp. 245-258). Amsterdam, Netherlands: Springer.

Spilchuk, B. (2009). Shila's Story of Teaching English/ESL in a Singapore Primary Neighbourhood School. Asian EFL Journal, 33. Retrieved June 10, 2012, from http://www.asian-efljournal.com/pta_Jan_09_bs.php

Tabak, I. (2004). Synergy: A complement to emerging patterns of distributed scaffolding. Journal of the Learning Sciences, 13(3), 305-335.

Wood, D., Bruner, J.S., \& Ross, G. (1976). The role of tutoring in problem solving. Journal of Child Psychology and Psychiatry, 17, 89-100.

Zhao, C. Liu, Y., \& Goh, H. (2007). Singaporean Chinese children's special Chinese vocabulary and their identity. In Proceedings of the Redesigning Pedagogy: Culture, Knowledge and Understanding Conference, Singapore, May 2007. Retrieved January 31, 2009, from http://conference.nie.edu.sg/2007/paper/papers/LAN226.pdf . 